\title{
Ultrasound Imaging for Tailored Treatment of Patients With Acute Shoulder Pain
}

Ramon P. G. Ottenbeijm, $M D^{1}$

Jochen W. L. Cals, MD, $P b D^{1}$

René Weijers, $M D, P b D^{2}$

Kurt Vanderdood, $M D^{3}$

Rob A. de Bie, PbD

Geert-Jan Dinant, MD, $P b D^{1}$

'Department of Family Medicine, CAPHRI School for Public Health and Primary Care, Maastricht University, Maastricht, The Netherlands

${ }^{2}$ Department of Radiology, Maastricht University Medical Centre, Maastricht, The Netherlands

${ }^{3}$ Department of Radiology, Orbis Medical Centre, Sittard-Geleen, The Netherlands

${ }^{4}$ Department of Epidemiology, CAPHRI School for Public Health and Primary Care, Maastricht University, Maastricht, The Netherlands

Conflicts of interest: authors report none.

\section{CORRESPONDING AUTHOR}

Ramon P. G. Ottenheijm, MD Department of Family Medicine CAPHRI School for Public Health and Primary Care

Maastricht University

PO BOX 616, 6200 MD

Maastricht, The Netherlands

ramon.ottenheijm@maastrichtuniversity.nl

\begin{abstract}
PURPOSE: The objective of this study was to assess the frequencies of ultrasound findings in patients with acute rotator cuff disorders in family medicine.

METHODS: In a prospective observational study, 129 patients aged 18 to 65 years with acute shoulder pain in whom the family physician suspected rotator cuff disease underwent ultrasound imaging.

RESULTS: Rotator cuff disease was present in $81 \%$ of the patients, and $50 \%$ of them had multiple disorders. Calcific tendonitis was the most frequently diagnosed specific disorder. An age of 40 years or older was most strongly related to rotator cuff disease.
\end{abstract}

CONCLUSIONS: Ultrasound imaging enables family physicians to rationalize treatment in nearly all patients who are aged 40 years and older with acute shoulder pain.

Ann Fam Med 2015;13:53-55. doi: 10.1370/afm.1734.

\section{INTRODUCTION}

$\Lambda$ lthough most patients with acute shoulder pain are cared for in family medicine, ${ }^{1}$ physical examinations used to evaluate the extensive spectrum of rotator cuff disease are often inadequate. ${ }^{2}$ As a result, recommended treatment for all patients in family medicine consists of stepwise treatment regardless of the specific underlying cause. ${ }^{3,4}$

The disadvantage of this management approach is that it may lead to unnecessary interventions and a delay in specific treatment, with subsequent prolonged recovery. Ultrasound imaging of the shoulder has become an accepted diagnostic modality for evaluating rotator cuff disorders, ${ }^{5}$ which potentially allows for more tailored treatment based on diagnostic subgroups. Against this background, it seemed useful to prospectively determine the frequencies of the disorders from the spectrum of rotator cuff disease in patients with shoulder pain in a family medicine setting. The objectives of this study were to assess prospectively the frequencies of specific findings as diagnosed using ultrasound imaging in patients with acute shoulder pain, and to identify possible predictors of shoulder pain.

\section{METHODS}

The present study was part of the Maastricht Ultrasound Shoulder Pain Trial (MUST) study, a randomized controlled trial among patients in family medicine with acute shoulder pain. The design of the MUST study has been presented in detail elsewhere. ${ }^{6}$ We included 129 patients with acute shoulder pain in whom the family physician suspected rotator cuff disease. Patients were eligible if they (1) had shoulder pain upon abduction with painful arc, (2) had symptoms having lasted for fewer than 3 months, (3) had no other episodes of shoulder pain in the previous 12 months, (4) were aged between 18 and 65 years.

All ultrasound examinations were obtained in real time using a Phillips IU22 (Phillips Medical Systems) with a 5-12 MHz broadband linear-array 
transducer (Phillips L12-5). All examinations were performed by experienced musculoskeletal radiologists at the Orbis Medical Centre, The Netherlands, using a protocol-based scanning approach. Standardized ultrasound diagnostic criteria for pathology were used. ${ }^{6}$ Ultrasound findings were prospectively evaluated for abnormalities of the rotator cuff, the long head of the biceps tendon, the subacromial-subdeltoid bursa, and the acromioclavicular joint, as well as for impingement.

We calculated means and standard deviations for continuous variables and percentages for categorical variables. Linear and logistic regression analyses were performed to determine the association of age, sex, and occupation with rotator cuff disorders in univariate analysis, followed by multivariate analysis, and expressed as odds ratios (ORs). Age was dichotomized at the most discriminating 5 -year age cutoff. $P$ $<.05$ was defined as statistically significant. Data were analyzed using IBM SPSS 21 (International Business Machines Corp).

Table 1. Frequencies of Ultrasound-Diagnosed Rotator Cuff Disorders and Predictive Factors ( $N=129$ Patients)

\begin{tabular}{|c|c|c|c|}
\hline Variable & $\mathrm{n} / \mathrm{n}(\%)$ & $\begin{array}{c}\text { Univariate Analysis } \\
\text { OR }(95 \% \mathrm{Cl})\end{array}$ & $P$ Value \\
\hline No disorder & 24/129 (18.6) & & \\
\hline Rotator cuff disease present & $105 / 129$ (81.4) & & \\
\hline Age $\geq 40$ y & $96 / 106$ (90.6) & $14.93(5.17-43.14)$ & $<.001$ \\
\hline Sex, female & $56 / 129(43.4)$ & $0.89(0.36-2.16)$ & .79 \\
\hline Occupational risk & $40 / 129(31.0)$ & $0.88(0.34-2.26)$ & .79 \\
\hline \multicolumn{4}{|l|}{ Specific disorder } \\
\hline Calcific tendonitis & $65 / 129(50.4)$ & & \\
\hline With other rotator cuff disorder & $39 / 65(60.0)$ & & \\
\hline Age $\geq 40$ y & $63 / 106(59.4)$ & $15.38(3.43-69.04)$ & $<.001^{\mathrm{b}}$ \\
\hline Tendinopathy & $37 / 129(28.7)$ & & \\
\hline With other rotator cuff disorder & 28/37 (75.7) & & \\
\hline Age $\geq 40$ y & $33 / 106(31.1)$ & $2.15(0.68-6.81)$ & $.19^{b}$ \\
\hline SASD bursitis & $26 / 129(20.2)$ & & \\
\hline With other rotator cuff disorder & $23 / 26(88.5)$ & & \\
\hline Age $\geq 40$ y & $26 / 106(24.5)$ & $\ldots{ }^{a}$ & $.004^{b}$ \\
\hline Partial-thickness tear & 25/129 (19.4) & & \\
\hline With other rotator cuff disorder & $16 / 25(64.0)$ & & \\
\hline Age $\geq 40$ y & $22 / 106(20.8)$ & $1.75(0.48-6.41)$ & $.56^{b}$ \\
\hline $\mathrm{ACJ}$-osteoarthritis & $16 / 129(12.4)$ & & \\
\hline With other rotator cuff disorder & $11 / 16(68.8)$ & & \\
\hline Age $\geq 40$ y & $16 / 106(15.1)$ & $\ldots^{a}$ & $.07^{b}$ \\
\hline Full-thickness tear & $4 / 129(3.1)$ & & \\
\hline With other rotator cuff disorder & $4 / 4(100)$ & & \\
\hline Age $\geq 40$ y & $4 / 106(3.8)$ & $\ldots^{a}$ & $>.99^{b}$ \\
\hline Impingement & $21 / 129(16.3)$ & & \\
\hline Age $\geq 40$ y & $20 / 106(18.9)$ & $5.12(0.65-40.23)$ & $.12^{b}$ \\
\hline \multicolumn{4}{|c|}{ Note: One patient had a biceps tendosynovitis. } \\
\hline \multicolumn{4}{|c|}{$\mathrm{ACJ}=$ acromioclavicular joint; $\mathrm{OR}$ = odds ratio; $\mathrm{SASD}=$ subacromial-subdeltoid. } \\
\hline \multicolumn{4}{|c|}{ a Odds ratio not provided; expected count in 1 of the cells is 0 . } \\
\hline
\end{tabular}

\section{RESULTS}

The mean age of the 129 included patients was 49.1 years $(\mathrm{SD}=10.6)$, and the dominant shoulder was affected in $54 \%$ (70). Table 1 displays the frequencies of rotator cuff disease, specific subgroups of the disease, and the associations for the subgroups. Impingement was always accompanied by a specific rotator cuff disorder. Fifty percent of the patients with rotator cuff disease (52) had multiple disorders. Table 2 displays the frequencies and combinations of multiple disorders observed. Linear regression analysis showed that the number of disorders significantly increased with increasing age $\left(R^{2}=0.29\right)$. The univariate analysis showed that age was significantly associated with the presence of rotator cuff disease, which was confirmed by the results of the multivariate analysis $(\mathrm{OR}=1.13 ; 95 \% \mathrm{CI}, 1.07-1.19)$.

\section{DISCUSSION}

Our results suggest that age of 40 years and older is a strong predictor of rotator cuff disease in patients complaining of acute shoulder pain in family medicine. More than $90 \%$ of patients with acute shoulder pain had 1 or more specific causes of rotator cuff disease detected on ultrasound imaging, with calcific tendonitis by far the most frequently observed.

Our study was the first to prospectively assess the findings of ultrasound imaging in patients visiting their family physician with acute shoulder pain. In comparison with studies in secondary care, our study showed a higher prevalence of calcific tendonitis and only a relatively small proportion of tears. ${ }^{5}$ These findings can be explained by the overrepresentation of patients in the diagnostic workup for surgery in secondary care populations.

Limitations of this study were that it lacked comparisons with the contralateral shoulder, reliability studies, a control group, and the relatively high number of participating radiologists $(n=13)$. The contralateral shoulder was scanned when findings were ambiguous, and $80 \%$ of all ultrasound procedures were performed by the 6 


\section{Table 2. Frequencies of Multiple Ultrasound- Diagnosed Disorders in Patients With Rotator Cuff Disease $(n=105)$}

\begin{tabular}{lc}
\hline No. and Combination of Disorders & No. (\%) \\
\hline 2 Disorders & $36(34.3)$ \\
Calcific tendonitis and tendinopathy & $13(12.4)$ \\
Calcific tendonitis and bursitis & $8(7.6)$ \\
Tendinopathy and bursitis & $3(2.9)$ \\
Tendinopathy and ACJ-osteoarthritis & $2(1.9)$ \\
Partial-thickness tear and calcific tendonitis & $2(1.9)$ \\
Partial-thickness tear and bursitis & $2(1.9)$ \\
Calcific tendonitis and ACJ-osteoarthritis & $2(1.9)$ \\
Full-thickness tear and calcific tendonitis & $1(0.9)$ \\
Full-thickness tear and bursitis & $1(0.9)$ \\
Full-thickness tear and ACJ-osteoarthritis & $1(0.9)$ \\
Bursitis and ACJ-osteoarthritis & $1(0.9)$ \\
3 Disorders & $14(13.3)$ \\
Partial-thickness tear, tendinopathy, and calcific & $6(5.7)$ \\
tendonitis & $2(1.9)$ \\
Partial-thickness tear, bursitis, and calcific tendonitis & $2(1.9)$ \\
Partial-thickness tear, bursitis, and tendinopathy & $2(1.9)$ \\
Calcific tendonitis, bursitis, and ACJ-osteoarthritis & $1(0.9)$ \\
Partial-thickness tear, bursitis, and ACJ-osteoarthritis & $1(0.9)$ \\
Full-thickness tear, calcific tendonitis, and & $2(1.9)$ \\
ACJ-osteoarthritis & $1(0.9)$ \\
4 Disorders & $1(0.9)$ \\
Calcific tendonitis, tendinopathy, bursitis, and & \\
partial-thickness tear & \\
Calcific tendonitis, tendinopathy, bursitis, and & \\
ACJ-osteoarthritis & \\
\hline ACJ = acromioclavicular joint. & \\
\hline & \\
\hline
\end{tabular}

most experienced of the musculoskeletal radiologists, reflecting common care practice in The Netherlands, which thus increases the generalizability of our results.

For daily practice, it is important that ultrasound findings should always be weighed against the clinical context, as asymptomatic findings may be observed. ${ }^{7}$ Our results show that ultrasound imaging enables family physicians to rationalize treatment, especially for patients aged 40 years and older. Importantly, it also provides an explanation for the complaints and informs about prognosis. For instance, calcific tendonitis is characterized by recurrent painful episodes. ${ }^{8}$ Given the low prevalence of full-thickness tears, unnecessary referrals to secondary care can be prevented. Patients with an inflammatory disorder, such as bursitis or calcifications in the resorption phase, may benefit from corticosteroid injections. In the case of multiple causes of rotator cuff disease, patients can be treated according to the disorder most likely to be symptomatic, or additional therapeutic approaches can be initiated.

The clinical value of ultrasound will depend on whether patient outcomes change. Further studies are needed to prove that early ultrasound imaging in the diagnostic workup improves patient outcome. ${ }^{6}$

To read or post commentaries in response to this article, see it online at http://www.annfammed.org/content/13/1/53.

Key words: shoulder joint; rotator cuff; shoulder impingement syndrome; ultrasound imaging; family practice

Submitted June 3, 2014; submitted, revised, September 16, 2014; accepted September 30, 2014.

Funding support: Medical Coordination Centre Omnes, Sittard-Geleen, The Netherlands, funded the ultrasound examinations.

Disclaimer: The Medical Coordination Centre Omnes, Sittard-Geleen was not involved in the design and conduct of the study; the collection, management, analysis, and interpretation of the data; or the preparation, review, and approval of the manuscript.

Acknowledgments: We would like to thank Jan Klerkx for linguistic advice.

\section{References}

1. van der Windt DA, Koes BW, Boeke AJ, Devillé W, De Jong BA, Bouter LM. Shoulder disorders in general practice: prognostic indicators of outcome. Br J Gen Pract. 1996;46(410):519-523.

2. Hanchard NC, Lenza M, Handoll HH, Takwoingi Y. Physical tests for shoulder impingements and local lesions of bursa, tendon or labrum that may accompany impingement. Cochrane Database Syst Rev. 2013;4:CD007427.

3. Clinical Knowledge Summeries NHS/NICE. Shoulder pain guideline. http://www.cks.nhs.uk/shoulder-pain. Jun 3, 2014.

4. Winters JC, van der Windt DAWM, Spinnewijn WEM, et al. Shoulder pain guideline of the Dutch College of General Practitioners [in Dutch]. Huisarts Wet. 2008;51(11):555-565.

5. Ottenheijm RPG, Jansen MJ, Staal JB, et al. Accuracy of diagnostic ultrasound in patients with suspected subacromial disorders: a systematic review and meta-analysis. Arch Phys Med Rehabil. 2010; 91(10):1616-1625

6. Ottenheijm RP, Joore MA, Walenkamp GH, et al. The Maastricht Ultrasound Shoulder pain Trial (MUST): ultrasound imaging as a diagnostic triage tool to improve management of patients with non-chronic shoulder pain in primary care. BMC Musculoskelet Disord. 2011;12:154.

7. Schibany N, Zehetgruber $\mathrm{H}$, Kainberger $\mathrm{F}$, et al. Rotator cuff tears in asymptomatic individuals: a clinical and ultrasonographic screening study. Eur J Radiol. 2004;51(3):263-268.

8. Carcia CR, Scibek JS. Causation and management of calcific tendonitis and periarthritis. Curr Opin Rheumatol. 2013;25(2):204-209. 